\title{
Welche Chancen bietet die Immunonkologie für ein indikationsübergreifendes Langzeitüberleben?
}

\author{
Lothar Bergmann $^{a} \quad$ Wolfram Brugger ${ }^{b}$ Wolfgang Herr ${ }^{c} \quad$ Andreas Mackensen $^{d}$ \\ Gabriele Multhoffe \\ ${ }^{a}$ Medizinischen Klinik II: Hämatologie/Onkologie, Universitätsklinikum Frankfurt, Deutschland; \\ ${ }^{b}$ Klinik für Innere Medizin II Hämatologie und Onkologie, Schwarzwald-Baar Klinikum, Villingen-Schwenningen, Deutschland; \\ ${ }^{c}$ Klinik und Poliklinik für Innere Medizin III, Universitätsklinikum Regensburg, Deutschland; \\ ${ }^{\mathrm{d}}$ Medizinische Klinik 5 - Hämatologie und Internistische Onkologie, Universitätsklinikum Erlangen, Deutschland; \\ ${ }^{e}$ Experimentelle RadioOnkologie und Strahlenbiologie, Technische Universität München, Deutschland
}

\section{Therapeutische Einsatzmöglichkeiten von Immun-Checkpoint-Inhibitoren}

\section{Andreas Mackensen}

Tumore nutzen verschiedene Escape-Mechanismen, um das körpereigene Immunsystem zu überlisten und sich gegenüber der Tumor-Immunabwehr durchzusetzen: Die Erkenntnis, dass sie immunregulative Moleküle wie z.B. CTLA-4 (Cytotoxic T Lymphocyte Antigen 4) oder PD-1 (programmed cell death 1) sowie deren Liganden PD-L1/PD-L2 instrumentalisieren können, um der Immunüberwachung $\mathrm{zu}$ entkommen (immune escape), hat einen vollständig neuen Therapiezugang für die Onkologie eröffnet [1].

Immun-Checkpoint-Moleküle wie CTLA-4 oder PD-1 werden auf Immunzellen als Rezeptoren exprimiert und übernehmen negative immunregulative Funktionen, um eine überschießende, Antigen-induzierte Aktivierung und Proliferation von T-Lymphozyten zu verhindern und die Selbsttoleranz aufrechtzuerhalten [2]. Beide Signalwege spielen für die potenzielle Immunevasion eines Tumors eine wichtige Rolle, da sie in ihrer natürlichen Funktion für die Induktion von distinkten immunsupprimierenden Mechanismen zuständig sind.

Während der Rezeptor CTLA-4 schon während der frühen Phase der Immunantwort (priming) für eine Limitierung der Autoimmunität durch T-Zellen zuständig ist, übernimmt PD-1 regulative Funktionen über die Effektor-T-Zellantwort, die sich vor allem im peripheren Gewebe, z.B. gegenüber einer Infektion, wahrscheinlich eher in der späten Phase der Immunantwort abspielen [3-5]. Tumorzellen können PD-1-Liganden wie PD-L1 hochregulieren und sich diesen Signalweg nutzbar machen, um sich vor einer antitumoralen Immunantwort zu schützen, die selbst noch in fortgeschrittenen Tumorstadien potenziell vorhanden ist [6]. Eine erhöhte PD-L1-Expression auf der Oberfläche von Tumorzellen wurde daher auch als prädiktiver Biomarker für anti-PD-1-gerichtete Therapieansätze diskutiert, zumal die PD-L1-Expression auf Tumorzellen in einigen Fällen mit der Prognose des Patienten korrelierte [7]. Der am besten geeignete Cut-off-Wert für PD-L1-Positivität konnte bis dato noch für keinen Tumor validiert werden. Auch die diagnostische Wertigkeit von PD-L1 als Biomarker wird derzeit in verschiedenen klinischen Studien untersucht [8-11].

Seitdem das Wirkprinzip einer immunvermittelten AntitumorStrategie durch die Checkpoint-Blockade erstmals beim fortgeschrittenen Melanom mit dem Antikörper Ipilimumab validiert wurde (siehe Kapitel «Rationale, Visionen und Grenzen der Immunonkologie»), ist das Interesse für weitere Checkpoint-Moleküle und ihre therapeutische Modulation sprunghaft angestiegen und damit auch die Hoffnung, dass diese Form der antitumoralen Behandlungsstrategie auch bei anderen Tumorentitäten effektiv sein könnte. Der immunbasierte Therapieansatz ist auch deshalb über verschiedene Indikationsbereiche hinweg von Interesse, da er ein enormes Anpassungspotenzial bietet, um sich auf die Heterogenität eines malignen Tumors einzustellen.

In einer Phase-I-Studie (CA209-003; CheckMate 003) konnten mit dem PD-1-Inhibitor Nivolumab (einem vollständig humanen, monoklonalen IgG4-Antikörper) sowohl bei Patienten mit einem weit fortgeschrittenen vorbehandelten malignen Melanom als auch bei verschiedenen Tumorentitäten wie dem nicht-kleinzelligen Lungenkarzinom (NSCLC) oder Nierenzellkarzinom (RCC) Tumorrückbildungen beobachtet werden [13]. Es folgten weitere erste klinische Studien, die mit anderen anti-PD-1/anti-PD-L1gerichteten Antikörpern wie z.B. Pembrolizumab bzw.

\section{KARGER}

Fax +497614520714 
Tab. 1. Immun-Checkpoint-Inhibition: Stand der klinischen Entwicklung mit dem Fokus auf zulassungsrelevante Studien

\begin{tabular}{|c|c|c|}
\hline Indikationsbereich & $\begin{array}{l}\text { Aktuell am weitesten fortgeschrittener } \\
\text { Stand der klinischen Entwicklung }\end{array}$ & Antikörper \\
\hline Malignes Melanom & $\begin{array}{l}\text { Ipilimumab zugelassen } \\
\text { Phase III - zulassungsrelevant }\end{array}$ & $\begin{array}{l}\text { anti-CTLA- } 4 \\
\text { anti-PD-1, anti-PD-L1; anti-CTLA- } 4+\text { anti-PD-1 }\end{array}$ \\
\hline Lungenkarzinom & Phase III - zulassungsrelevant & anti-PD-1; anti-PD-L1; anti-CTLA-4 \\
\hline Nierenzellkarzinom & Phase III - zulassungsrelevant & anti PD-1; anti-CTLA-4 + anti-PD-1 \\
\hline Prostatakarzinom & Phase III - zulassungsrelevant & anti-CTLA-4 \\
\hline Glioblastom & Phase III - zulassungsrelevant & anti-PD-1; anti-PD-L1 \\
\hline Kopf-Hals-Tumore & Phase III - zulassungsrelevant & anti-PD-1 \\
\hline $\begin{array}{l}\text { Hämatologische Neoplasien } \\
\text { (z.B. Hodgkin-Lymphom, Non-Hodgkin-Lymphom, } \\
\text { Multiples Myelom) }\end{array}$ & Phase II / III - zulassungsrelevant & anti-PD-1; anti-CTLA-4 \\
\hline
\end{tabular}

MPDL3280A bei Patienten mit fortgeschrittenen soliden Tumorentitäten durchgeführt wurden $[14,15]$.

\section{Heterogenität des Tumors als Chance?}

Die Wirksamkeit über multiple Tumortypen hinweg impliziert einen möglichen Zusammenhang zwischen der genetischen Vielfalt des Tumorgenoms und der «Immunogenität» eines Tumors: So fällt auf, dass die immunvermittelte Therapie mit Checkpoint-Inhibitoren insbesondere bei Tumoren, die eine hohe genetische Heterogenität aufweisen, in klinischen Studien erste ermutigende Ergebnisse zeigt [16, 17].

Beim Melanom und NSCLC handelt es sich zudem um 2 Tumortypen, die häufig über Karzinogene wie UV-Licht bzw. Tabakrauch ausgelöst werden und die höchsten somatischen Mutationsraten im Tumorgenom aufweisen [18]. Dabei kodieren die meisten der somatischen Tumormutationen für intrazelluläre Proteine, von denen kurze Peptidfragmente durch ein MHC-Molekül auf der Tumorzelloberfläche präsentiert werden: Theoretisch könnten diese Neoantigene die Aufmerksamkeit tumorspezifischer T-Zellen auf sich ziehen und eine antitumorale Immunreaktion hervorrufen [16]. Gleichzeitig ist das adaptive Immunsystem mit einer hohen Plastizität ausgestattet: Da es sich laufend anpassen kann, würde ein weites Neoantigenspektrum unter einer Immun-Checkpoint-Blockade möglicherweise auch die Entwicklung eines besonders breiten T-Zell-Repertoires induzieren und gegenüber der individuellen, subklonalen Heterogenität des Tumors bestehen können $[16,19]$.

Die hohe inter- und intratumorale Heterogenität eines TumorGenoms erklärt auch, warum es so schwierig ist, für multiple Tumorerkrankungen einheitlich effektive Therapieoptionen zu entwickeln $[18,20]$ : Beispielsweise ist die Anzahl der Onkogene, die für das Tumorwachstum relevant sind und über die z.B. die zielgerichtete, pharmakologische Hemmung von Tyrosinkinasen adressierbar ist, limitiert - ebenso wie die Dauer der darüber erzielbaren klinischen Remission [21, 22]. Auch können fortgeschrittene Tumore mit der Zeit Resistenzen entwickeln, indem sie auf andere onkogene Signalwege ausweichen [21, 22].

Somit wird künftig zunehmend die Frage aufkommen, inwieweit zielgerichtete Therapien wie Tyrosinkinase-Inhibitoren (TKI) auf tumorassoziierte Mutationen einen selektiven Druck ausüben und dadurch das Therapieansprechen auf immunmodulierende Strategien beeinflussen könnten [23]. Nach den bisherigen Erfahrungen mit immunonkologisch aktiven Substanzen deutet vieles darauf hin, dass deren Einsatz unter anderem nicht an das Vorhandensein von Treibermutationen gebunden ist bzw. unabhängig vom histologischen Subtyp eines Tumors sein könnte. Ein breiter Behandlungsansatz erscheint möglich (Tab. 1).

\section{Immunonkologie beim NSCLC - aktuelle Perspektiven}

\section{Wolfram Brugger}

Das Lungenkarzinom repräsentiert einen Tumor mit nach wie vor sehr ungünstiger Prognose und der in Deutschland mit Abstand häufigsten Todesursache bei Männern und dritthäufigsten bei Frauen, die auf eine maligne Tumorerkrankung zurückgehführt werden kann [24]. Dabei macht das nicht-kleinzellige Lungenkarzinom (NSCLC) einen Anteil von etwa 85\% aller Lungenkarzinome aus [25]. Die Mehrheit der Tumore ist bei Erstdiagnose bereits lokal fortgeschritten oder metastasiert, und das Gesamtüberleben beim fortgeschrittenen NSCLC ist sehr gering: Die relativen 5-Jahres-Überlebensraten hängen vom Stadium der Erkrankung bei Erstdiagnose ab und betragen in der Regel etwa 15\% [25].

Histologisch wird das Lungenkarzinom in die Gruppe der NSCLC mit dem Plattenepithelkarzinom und dem Nicht-Plattenepithelkarzinom (vor allem Adenokarzinom und großzelliges Karzinom) eingeteilt. Die therapeutischen Möglichkeiten haben sich insbesondere beim Adenokarzinom der Lunge durch die Identifizierung von onkogenen Treibermutationen und die Einführung von zielgerichteten Therapien signifikant verbessert. Allerdings ist die genetisch definierte Subgruppe mit aktivierender EGFR-Mutation bzw. Translokation des ALK-Onkogens auf einen kleinen Teil der Patienten beschränkt, d.h. je nach Studie auf maximal 15-20\% aller NSCLC-Patienten [26]. 
Nach bisheriger Auffassung galt das NSCLC bislang als «nichtimmunogener» Tumor [27]. Die klinische Aktivität, die sich schon in den ersten frühen klinischen Studien unter einer anti-PD-1-gericheteten Checkpoint-Blockade abzeichnete, wurde daher als Überraschung gewertet $[13,27,28]$. Inzwischen befinden sich zahlreiche Substanzen beim Lungenkarzinom in Entwicklung, die sich gegen den PD-1/PD-L1-assoziierten Signalweg richten (z.B. Nivolumab, Pembrolizumab, MPDL3280A, MedI-4736) [27].

Die Hypothese, dass die erhöhte genetische Heterogenität eines Tumors eine Schlüsselrolle für das immunvermittelte Ansprechen spielen könnte, wird durch die Beobachtung bestärkt, dass ehemalige Raucher sowie Raucher gegenüber jenen, die nie geraucht hatten, eine bessere Gesamtansprechrate unter einer anti-PD-L1-gerichteten Checkpoint-Blockade zeigten [29]. In einer Phase-Ib-Studie mit Nivolumab schnitten NSCLC-Patienten, die ehemalige Raucher ( $>5$ Packungsjahre) waren bzw. immer noch Raucher sind, gegenüber Patienten mit $<5$ Packungsjahren (Wenig-Raucher) sowie jenen, die nie geraucht hatten, ebenfalls mit einem signifikant besseren Therapieansprechen ab [30].

Dazu passt die Beobachtung, dass NSCLC-Patienten, die zuvor geraucht haben bzw. Raucher sind im Gegensatz zu NSCLC-Patienten, die nie geraucht hatten, deutlich höhere mediane Mutationsraten aufweisen: Tumortypen, die nur ein eingeschränktes Ansprechen auf anti-PD-1/PD-L1-inhibitorische Therapiestrategien entwickelten, sind hingegen mit relativ niedrigeren medianen $\mathrm{Mu}-$ tationsraten assoziiert [31].

Überdies profitierten NSCLC-Patienten nicht nur unabhängig vom histologischen Subtyp (Platten- vs. Nicht-Plattenepithelkarzinom) von einer Anti-PD-1-Blockade, sondern auch unabhängig von der Anzahl der vorangehenden Therapien (3 oder $\geq$ Vortherapien), dem Vorliegen einer EGFR- oder KRAS-Mutation oder dem PD-L1-Tumorstatus [32-34].

Basierend auf den Phase-I-Daten (CheckMate-003, CA209003) und Phase-II-Daten (CheckMate-063, CA209-063) wurde beim NSCLC ein breites Phase-III-Studienprogramm mit dem humanen, monoklonalen Antikörper Nivolumab in verschiedenen Therapielinien initiiert:

- In der Phase-III-Studie CheckMate 026 (CA209-026) wird der PD-1-Inhibitor Nivolumab mit einer Erstlinien-Chemotherapie (Nivolumab vs. Chemotherapie der Wahl) bei nicht vorbehandelten Patienten mit fortgeschrittenem, PD-L1-positivem NSCLC verglichen. Primärer Endpunkt der Studie ist das progressionsfreie Überleben nach RECIST (response evaluation criteria in solid tumors).

- In der Phase-III-Studie CheckMate 057 (CA209-057) werden vorbehandelte Patienten mit fortgeschrittenem (nicht-resezierbarem) oder metastasiertem Nicht-Plattenepithelkarzinom der Lunge entweder mit Nivolumab oder Docetaxel behandelt. Primärer Studienendpunkt ist das Gesamtüberleben (overall survival, OS).

- In der Phase-III-Studie CheckMate-017 (CA209-017) wurden vorbehandelte Patienten mit fortgeschrittenen (nicht-resezierbarem) oder metastasierten nicht-kleinzelligen Plattenepithelkarzinomen der Lunge nach einem Therapieversagen auf eine platinbasierte Erstlinien-Doublet-Chemotherapie entweder mit
Nivolumab oder Docetaxel behandelt. Primärer Studienendpunkt war das Gesamtüberleben (OS). Die Studie wurde vorzeitig beendet, als das Data-Monitoring-Komitee feststellte, dass der Studienendpunkt frühzeitig erreicht worden war: Patienten, die mit Nivolumab behandelt wurden, zeigten gegenüber dem Kontrollarm ein überlegenes OS [35]. In den USA wurde der PD-1-Inhibitor Nivolumab beim squamösen NSCLC in Zweitlinie bereits durch die FDA zugelassen, in Europa ist eine Zulassung bei der EMA beantragt (www.bms.com/news/press_releases).

\section{Immunonkologie beim RCC -} ein Revival mit Durchbruch?

\section{Lothar Bergmann}

Historisch zählt das Nierenzellkarzinom (renal cell cancer, RCC) neben dem malignen Melanom ebenfalls zu den klassischen, als «immunogen» eingestuften Tumoren: Ähnlich wie beim Melanom wurden auch beim RCC Spontanremissionen beobachtet. Im Gegensatz zum NSCLC wurden immunvermittelte Therapieansätze beim Nierenzellkarzinom bereits vor Jahrzehnten erprobt und eingeführt [36, 37].

$\mathrm{Zu}$ einer deutlichen Verbesserung der Prognose (verlängertes progressionsfreies Überleben) kam es allerdings erst mit der Etablierung von anti-angiogenetisch wirksamen Tyrosinkinase- sowie mTOR-Inhibitoren im Therapiealgorithmus des metastasierten RCC [38-40]. Stratifiziert nach den Risiken für eine günstige, mittlere bzw. schlechte Prognose nach IMDC (International Metastatic Renal Cell Carcinoma Database Consortium) liegt die mediane Gesamtüberlebenszeit nach einer zielgerichteten Therapie in Zweitlinie beim metastasierten RCC bei 35,3 bzw. 16,6 und 5,4 Monaten [38]. Da die Erkrankung nach wie vor nicht kurativ behandelbar ist, werden alternative Therapieoptionen gesucht, die zu einer Verlängerung der Überlebenszeit führen.

Mit der Identifizierung der ersten immunregulatorisch relevanten Checkpoint-Signalwege, die der Tumor im Sinn eines «immune-escape»-Mechanismus gegen die T-Zell-vermittelte Immunüberwachung nutzen kann, lebte das Prinzip eines immunvermittelten Therapieansatzes beim metastasierten RCC (metatstatic RCC, mRCC) wieder auf: Ermutigt durch die ersten, frühen klinischen Daten der PD-1-Inhibition mit Nivolumab sowie der PD-L1Inhibition mit MPDL3280A bei stark vorbehandelten, fortgeschrittenen RCC-Patienten [41, 42], wurden auch beim mRCC breite klinische Entwicklungsprogramme mit mehreren anti-PD-1-/ PD-L1-gerichteten monoklonalen Antikörpern auf den Weg gebracht (unter anderem Nivolumab, Pembrolizumab, MPDL3280A, MedI-4736). Dabei war Nivolumab der erste PD-1-Inhibitor, der RCC-spezifisch in klinischen Studien evaluiert wurde [13].

Die ersten Phase-II-Daten für einen PD-1-Inhibitor beim RCC lieferte die Studie CheckMate-010 (CA209-010): Vorbehandelte, therapierefraktäre Patienten mit einem mRCC, die bereits 1-3 systemische Vortherapien erhalten hatten, darunter mindestens eine antiangiogenetische Therapie, könnten unter der Monotherapie 

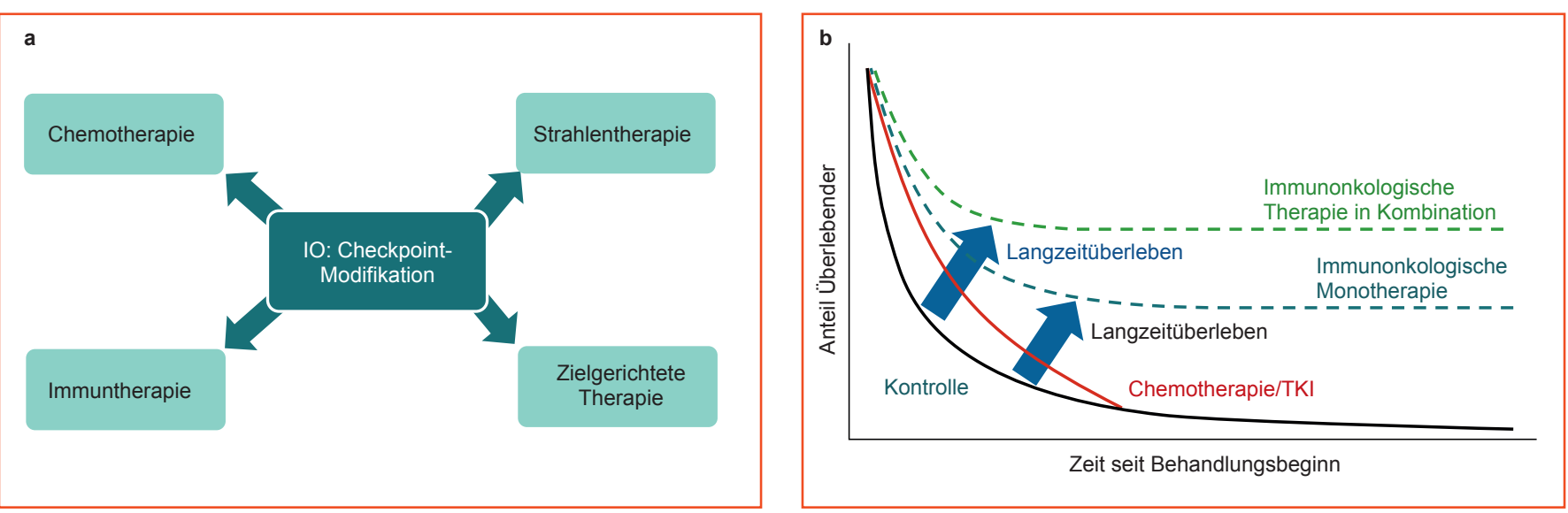

Abb. 1. Potenzielle Synergieeffekte: hypothetisches Potenzial der Immun-Checkpoint-Blockade in Kombination mit anderen Therapien (modifiziert nach Ott et al. [53]); (a) Immunonkologische Ansätze zur Optimierung des klinischen Benefits [54-57]; (b) Vision: Dauerhaftigkeit des Ansprechens. Das Potenzial der Immunonkologie.

mit Nivolumab von längerfristigen Ansprechraten profitieren - bei einem akzeptablen Verträglichkeitsprofil [43]. Die Frage, inwieweit die Wirksamkeit einer Checkpoint-Modifier-Therapie durch eine PD-L1-Expression des Tumors prädizierbar ist, wurde ebenfalls mit untersucht, lieferte aber keine Hinweise auf einen eindeutigen Zusammenhang.

Vor dem Hintergrund der ermutigenden Phase-II-Daten wird der PD-1-Inhibitor Nivolumab derzeit in 2 großen, randomisierten und zulassungsrelevanten Studien evaluiert:

- In der Studie CheckMate-025 (CA209-025) werden vorbehandelte ( $\mathrm{n}=822$ ) Patienten (mindestens eine, maximal 2 Vortherapien mit einem TKI) mit einem fortgeschrittenen oder metastasierten Klarzell-RCC entweder auf den PD-1-Inhibitor Nivolumab oder Everolimus randomisiert. Primärer Studienendpunkt ist das mediane Gesamtüberleben (mOS). Die Rekrutierung der Patienten ist bereits abgeschlossen.

- Für die Erstlinientherapie mit Nivolumab in Kombination mit Ipilimumab versus Sunitinib in Monotherapie werden derzeit Patienten für die Teilnahme an CheckMate-214 (CA209-214) rekrutiert (geplant $\mathrm{n}=1070$ ), die ein fortgeschrittenes oder metastasiertes RCC aufweisen und noch keine Vortherapie erhalten haben: Co-primäre Outcome-Parameter sind das mediane progressionsfreie Überleben sowie das mOS.

\section{Immunonkologie in der Hämatologie - integrierte Therapiekonzepte für nachhaltige Tumortherapie}

\section{Wolfgang Herr, Gabriele Multhoff}

Das Thema Immun-Checkpoint-Inhibition ist in der Hämatoonkologie noch verhältnismäßig jung: Die Mehrheit der klinischen Daten bei hämatologischen Tumoren stammt aus Phase-IStudien. Auf die potenzielle Bedeutung der PD-1/PD-L1-Achse für lymphoproliferative Erkrankungen gibt es aber bereits eine ganze Reihe von Hinweisen: Eine erhöhte PD-L1- bzw. PD-1-Expression wurde unter anderem beim diffus-großzelligen B-Zell-Lymphom und beim follikulären B-Zell-Non-Hodgkin-Lymphom gemessen; eine erhöhte PD-1/PD-L1-Expression konnte auch bei der chronisch lymphatischen Leukämie festgestellt werden [44-46].

Einen ersten klinischen Ausblick boten die kürzlich publizierten Phase-I-Daten zum PD-1-Inhibitor Nivolumab beim klassischen, rezidivierten oder therapierefraktären Hodgkin-Lymphom (HL) [47], das derzeit auch in einer laufenden zulassungsrelevanten Phase-II-Studie untersucht wird sowie die ersten Phase-Ib-Daten zum PD-1-Inhibitor Pembrolizumab - ebenfalls beim klassischen HL [48]. Das klassische HL ist vermutlich durch eine genetisch determinierte Vulnerabilität für eine PD-1-Inhibition charakterisiert (vermehrte Expression von PD-L1/PD-L2 bei allen behandelten HL-Patienten infolge einer genetischen Alteration auf Chromosom 9 (9p24.1)) [47, 49].

Perspektivisch sind in der Hämatologie auch integrierte, multimodale Therapieansätze denkbar, um über die Einbeziehung von konventionellen Therapieoptionen eine effektive Reduktion der Tumormasse zu erreichen und anschließend über die immunonkologische Therapie eine potenziell nachhaltige, lang anhaltende Immunresponse einzuleiten.

\section{Potenzierung von Immuneffekten bei konventioneller Therapie}

Konventionelle onkologische Therapieoptionen wie sie in erster Linie von der Radio- oder Chemotherapie sowie auch molekularen, zielgerichteten Therapieansätzen repräsentiert werden, verfügen nicht nur über direkt zytotoxische, sondern auch über bislang weniger prominente, immunmodulatorische Eigenschaften.

Beispielweise wurden unter einer Bestrahlung oder Chemotherapie neben den direkt zytotoxischen Effekten, die über DNA-Schäden mediiert werden, auch schon seit längerem Phänomene beschrieben, die zu einer Potenzierung der antitumoralen Immunabwehr beitragen können: So können strahleninduzierte Schäden an Tumorzellen unter anderem die verstärkte Membranexpression von MHC-Klasse-I- und MHC-Klasse-II-Molekülen, Fas-Liganden, Danger-Signalen wie z.B. HMBG1 und Heat Shock Protein 70 
(Hsp70) oder des interzellulären Adhäsionsmoleküls-1 auf Endothelzellen bewirken: diskutiert als möglicher Beitrag für eine Toder NK-Zell-vermittelte antitumorale Immunantwort bzw. den Aufbau einer antitumoralen Immunität [50, 51].

Darüber hinaus sind strahlungsbiologische Mechanismen beschrieben, die nicht auf die Tumorlokalität bzw. die getroffenen Zellen oder das Gewebe beschränkt sind, sondern im Rahmen von Bystander-Effekten auch abskopale (ab-scopus = jenseits vom Zielfokus) Therapieeffekte haben können [52]. Damit keimt die Hoffnung auf, dass kombinierte Therapiekonzepte für eine komplementäre oder sogar auch synergistische Verstärkung der T-Zell-vermittelten, antitumoralen Immunreaktion auf lange Sicht infrage kommen und den Anteil der fortgeschrittenen Tumorpatienten weiter erhöhen könnten, die unter einer-Checkpoint-Inhibitor-Therapie von einem hemmenden Effekt auf den Tumorprogress sowie verbesserten Langzeitüberleben profitieren (Abb. 1).

\section{Disclosure Statement}

Die Autoren waren Referenten beim «5. Interdisziplinären Expertenforum», das von Bristol-Myers Squibb unterstützt wurde.

\section{Kernpunkte}

- Der immunvermittelte Therapieansatz über die CheckpointInhibition ermöglicht einen Angriff auf den Tumor, indem er das endogene Potenzial eines T-/NK-Zell-vermittelten, antitumoralen Immuneffekts potenziert.

- Da das adaptive Immunsystem im Zentrum der Therapieantwort steht und eine tumorindividuelle Anpassung ermöglicht, ist die Checkpoint-Inhibition weitestgehend unabhängig vom Mutationsstatus oder dem histologischen Subtyp des Tumors sowie der Art und Anzahl der Vortherapien.

- Am weitesten fortgeschritten ist die klinische Entwicklung der Checkpoint-Blockade in den Indikationsbereichen Melanom, NSCLC und RCC. Die bisherige Datenlage zur antiPD-1/PD-L1-gerichteten Checkpoint-Blockade lässt auf erhöhte Raten an günstigen Langzeitverläufen hoffen.

- Neben der klinischen Prüfung bei zahlreichen soliden Tumoren werden immunonkologische Therapieansätze auch im Rahmen von klinischen Studien in der Hämatoonkologie untersucht.

\section{Literatur}

1 Hanahan D, Weinberg RA: Hallmarks of cancer: the next generation. Cell 2011;144:646-674.

2 Vigano S, Perreau M, Pantaleo G, Harari A: Positive and negative regulation of cellular immune responses in physiologic conditions and diseases. Clin Dev Immunol 2012;2012:485781.

$\checkmark 3$ Topalian SL, Drake CG, Pardoll DM: Targeting the PD-1/B7-H1(PD-L1) pathway to activate anti-tumor immunity. Curr Opin Immunol 2012;24:207-212.

4 Pardoll DM: The blockade of immune checkpoints in cancer immunotherapy. Nat Rev Cancer 2012;12:252264.

5 Iwai Y, Ishida M, Tanaka Y, et al.: Involvement of PD-L1 on tumor cells in the escape from host immune system and tumor immunotherapy by PD-L1 blockade. Proc Natl Acad Sci USA 2002;99:12293-7.

6 Pardoll DM, Drake C: Immunotherapy earns its spot in the ranks of cancer therapy. J Exp Med 2012;209: 201-209.

7 Dong H, Strome SE, Salomao DR, et al.: Tumor-associated B7-H1 promotes T-cell apoptosis: a potential mechanism of immune evasion. Nat Med 2002;8:793800 .

8 Mahoney KM, Atkins MB: Prognostic and predictive markers for the new immunotherapies. Oncology (Williston Park) 2014;28(suppl 3);pii:202335.

9 Brahmer JR, Horn L, Gandhi L, et al.: Nivolumab (anti-PD-1, BMS-936558, ONO-4538) in patients (pts) with advanced non-small-cell lung cancer (NSCLC): survival and clinical activity by subgroup analysis. J Clin Oncol 2014;32(suppl):abstr 8112.
10 Soria J, Gettinger S, Gordon MS, et al.: Biomarkers associated with clinical activity of PD-L1 blockade in non-small cell lung cancer (NSCLC) patients (pts) in a Phase I study of MPDL3280A. Annals of Oncology 2014;25(Suppl 4):iv426-iv470. 10.1093/annonc/mdu349.

11 Gandhi L, Balmanoukian A, Hui R, et al.: MK-3475 (anti-PD-1 monoclonal antibody) for non-small cell lung cancer (NSCLC): antitumor activity and association with tumor PD-L1 expression. AACR Annual Meeting 2014;San Diego.Abstract CT105.

12 Massari F, Santoni M, Ciccarese C, et al.: PD-1 blockade therapy in renal cell carcinoma: current studies and future promises. Cancer Treat Rev 2015;41:114121.

13 Brahmer JR, Drake CG, Wollner I, et al.: Phase I study of single-agent anti-programmed death-1 (MDX$1106)$ in refractory solid tumors: safety, clinical activity, pharmacodynamics, and immunologic correlates. J Clin Oncol 2010;28:3167-3175.

14 Herbst RS, Gordon MS, Fine GD, et al.: A study of MPDL3280A, an engineered PD-L1 antibody in patients with locally advanced or metastatic tumors. J Clin Oncol 2013;31(suppl 15):3000.

15 Patnaik A, Kang SP, Tolcher AW, et al.: Phase I study of MK-3475 (anti-PD-1 monoclonal antibody) in patients with advanced solid tumors. J Clin Oncol 2012; 30(suppl 15): 2512.

16 Brown SD, Warren RL, Gibb EA, et al.: Neo-antigens predicted by tumor genome meta-analysis correlate with increased patient survival. Genome Res 2014;24: $743-750$.
7 Rizvi NA, Hellmann MD, Snyder A, et al.: Mutational landscape determines sensitivity to PD-1 blockade in non-small cell lung cancer. Sciencemag 2015;10.1126/ science.aaal 348

18 Alexandrov LB, Nik-Zainal S, Wedge DC, et al.: Signatures of mutational processes in human cancer. Nature 2013;500:415-450.

19 Heemskerk B, Kvistborg P, Schumacher TNM: The cancer antigenome. EMBO J 2013;32:194-203.

20 Vogelstein B, Papadopoulos N, Velculescu VE, et al.: Cancer genome landscapes. Science 2013;339:15461558.

21 Kwak EL, Bang YJ, Camidge DR, et al.: Anaplastic lymphoma kinase inhibition in non-small-cell lung cancer. N Engl J Med 2010;363:1693-703.

22 Rosell R, Moran T, Carcereny E, et al.: Non-small-cell lung cancer harbouring in the EGFR kinase domain. Clin Transl Oncol 2010;12:75-80.

23 Boni A, Cogdill AP, Dang P, et al.: Selective BRAFV600E inhibition enhances T-cell recognition of melanoma without affecting lymphocyte function. Cancer Res 2010;70:5213-5219.

24 Robert Koch-Institut: Krebsarten, Lungenkrebs. $w w w$. rki.de/Krebs/DE/Content/Krebsarten/Lungenkrebs/lungenkrebs_node.html (Zugriff 04.03.2015).

25 Molina JR, Yang P, Cassivi SD, et al.: Non-small cell lung cancer: epidemiology, risk factors, treatment, and survivorship. Mayo Clin Proc 2008;83:584-594.

26 Pao W, Chmielecki J: Rational, biologically based treatment of EGFR-mutant non-small-cell lung cancer. Nat Rev Cancer 2010;10:760-774. 
27 Helissey C, Champiat S, Soria JC: Immune checkpoint inhibitors in advanced nonsmall cell lung cancer. Curr Opin Oncol 2015;27:108-117.

28 Topalian SL, Hodi FS, Brahmer JR, et al.: Safety, activity, and immune correlates of anti-PD-1 antibody in cancer. N Engl J Med 2012;366:2443-2454.

29 Horn L, Herbst RS, Spigel D, et al.: An analysis of the relationship of clinical activity to baseline EGFR status, PD-L1 expression and prior treatment history in patients with non-small cell lung cancer (NSCLC) following PD-L1 blockade with MPDL3280A (Anti-PDL1). J Thor Oncol 2013;8(suppl 2):S364

30 Hellmann BC, Creelan K, Woo K, et al.: Smoking history and response to nivolumab in patients with advanced NSCLCs. Ann Oncol 2014;25(suppl 4):iv426-iv470.

31 Champiat S, Ferté C, Lebel-Binay S, et al.: Bridging mutational load and immune checkpoints efficacy. OncoImmunology 2014;3:e27817.

32 Antonia SJ, Grosso JF, Horak CE: Association of tumor PD-L1 expression and immune biomarkers with clinical activity in patients with non-small cell lung cancer (NSCLC) treated with nivolumab (antiPD-1; BMS-936558; ONO-4538). Thorac Oncol 2013; 8(suppl 2):p2.11-035.

33 Gettinger S, Horn L, Antonia SJ, et al.:Efficacy of nivolumab (anti-PD-1; BMS-936558; ONO-4538) in patients with previously treated advanced non-small cell lung cancer (NSCLC): subpopulation response analysis in a phase 1 trial. Thorac Oncol 2013;8(suppl 2):P2.11-038.

34 Brahmer JR, Horn L, Gandhi L, et al.: Nivolumab (anti-PD-1, BMS-936558, ONO-4538) in patients (pts) with advanced non-small cell lung cancer (NSCLC): survival and clinical activity by subgroup analysis. Clin Oncol 2014;32:abstr 8112^.

35 Bristol-Myers Squibb: CheckMate -017, A Phase 3 Study of Opdivo (Nivolumab) Compared to Docetaxel in Patients with Second-Line Squamous Cell Nonsmall Cell Lung Cancer, Stopped Early; Pressemitteilung vom 11.01.2015. http://news.bms.com/pressrelease/checkmate-017-phase-3-study-opdivonivolumab-compared-docetaxel-patients-second-line-s (Zugriff 04.03.2015).

36 Yang JC, Childs R: Immunotherapy for renal cancer. J Clin Oncol 2006;24:5576-5583.
Coppin C, Porzsolt F, Awa A, et al.: Immunotherapy for advanced renal cell cancer. Cochrane Database Syst Rev 2005;25:CD001425.

38 Ko JJ, Xie W, Kroeger N, et al.: The International Metastatic Renal Cell Carcinoma Database Consortium model as a prognostic tool in patients with metastatic renal cell carcinoma previously treated with first-line targeted therapy: a population-based study. Lancet Oncol 2015;16:293-300.

39 Bergmann L, Beck J, Bothe K, Brinkmann OA, et al.: Treatment algorithm for metastatic renal cell carcinoma-recommendations based on evidence and clinical practice. Oncol Res Treat 2014;37:136-141.

40 Bergmann L, Maute L, Guschmann M: Temsirolimus for advanced renal cell carcinoma. Expert Rev Anticancer Ther 2014;14:9-21.

41 Topalian SL, Sznol M, Brahmer JR, et al.: Nivolumab (anti-PD-1; BMS-936558;ONO-4538) in patients with advanced solid tumors: survival and long-term safety in a phase I trial. J Clin Oncol. 2013;31(suppl):abstr 3002.

42 Cho DC, Sosman JA, Mario Sznol M, et al.: Clinical activity, safety, and biomarkers of MPDL3280A, an engineered PD-L1 antibody in patients with metastatic renal cell carcinoma (mRCC). J Clin Oncol. 2013; 31(suppl):abstr 4505.

43 Motzer RJ, Rini BI, McDermott DF: Nivolumab for metastatic renal cell carcinoma (mRCC): results of a randomized, dose-ranging phase II trial. J Clin Oncol 2014;32(suppl):abstr 5009.

44 Andorsky DJ, Yamada RE, Said J, et al.: Programmed death ligand 1 is expressed by non-Hodgkin lymphomas and inhibits the activity of tumor-associated $\mathrm{T}$ cells. Clin Cancer Res 2011;17:4232-4244.

45 Richendollar BG, Pohlman B, Elson P, Hsi ED: Follicular programmed death-1-positive lymphocytes in the tumor microenvironment are an independent prognostic factor in follicular lymphoma. Hum Pathol 2011;42:552-557.

46 Brusa D, Serra S, Coscia M, et al.: The PD-1/PD-L1 axis contributes to T-cell dysfunction in chronic lymphocytic leukemia. Haematologica 2013;98:953-963.

47 Ansell SM, Lesokhin AM, Borrello I, et al.: PD-1 Blockade with Nivolumab in relapsed or refractory Hodgkin's lymphoma. N Engl J Med 2015;372:311319.
48 Moskowitz CH, Ribrag V, Michot JM, et al.: PD-1 blockade with the monoclonal antibody pembrolizumab (MK-3475) in patients with classical Hodgkin lymphoma after brentuximab vedotin failure: preliminary results from a phase $1 \mathrm{~b}$ study (KEYNOTE-013). 56th Ash Annual Meeting and Exposition 2014;oral abstract presentation no. 290. https://ash.confex.com/ ash/2014/webprogram/Paper75615.html (Zugriff 04.03.2015).

49 Armand P, Ansell SM, Lesokhin AM, et al.: Nivolumab in patients with relapsed or refractory Hodgkin lymphoma - preliminary safety, efficacy and biomarker results of a phase I study. 56th Ash Annual Meeting and Exposition 2014;oral abstract presentation no. 289. https://ash.confex.com/ash/2014/webprogram/ Paper73050.html (Zugriff 04.03.2015).

50 Garnett T, Palena C, Chakraborty M, et al.: Sublethal irradiation of human tumor cells modulates phenotype resulting in enhanced killing by cytotoxic T lymphocytes. Cancer Res 2004;64:7985-7994.

51 Reits EA, Hodge JW, Herberts CA, et al.: Radiation modulates the peptide repertoire, enhances MHC class I expression, and induces successful antitumor immunotherapy. J Exp Med 2006;203:1259-1271.

52 Wattenberg MM, Fahim A, Ahmed MM, et al.: Unlocking the combination: potentiation of radiation-induced antitumor responses with immunotherapy. Radiat Res 2014;182:126-138.

53 Ott PA, Hodi FS, Robert C: CTLA-4 and PD-1/PD-L1 blockade: new immunotherapeutic modalities with durable clinical benefit in melanoma patients. Clin Cancer Res 2013;19:5300-5309.

54 Drake CG: Combination immunotherapy approaches. Ann Oncol 2012;23(suppl 8):viii41-viii46.

55 Hannani D, Sistigu A, Kepp O, et al.: Prerequisites for the antitumor vaccine-like effect of chemotherapy and radiotherapy. Cancer J 2011;17:351-358.

56 Ménard C, Martin F, Apetoh L, et al.: Cancer chemotherapy: not only a direct cytotoxic effect, but also an adjuvant for antitumor immunity. Cancer Immunol Immunother 2008;57:1579-1587.

57 Ribas A, Wolchok JD: Combining cancer immunotherapy and targeted therapy. Curr Opin Immunol 2013;25:291-296. 